\title{
Signal enhancement in amperometric peroxide detection by using graphene materials with low number of defects
}

\author{
Alexander Zöpfl ${ }^{1}$ - Masoumeh Sisakthi ${ }^{2}$. Jonathan Eroms ${ }^{2}$ • Frank-Michael Matysik ${ }^{1}$. \\ Christoph Strunk ${ }^{2}$. Thomas Hirsch ${ }^{1}$
}

Received: 18 June 2015 / Accepted: 8 August 2015 / Published online: 15 August 2015

(C) Springer-Verlag Wien 2015

\begin{abstract}
Two-dimensional carbon nanomaterials ranging from single-layer graphene to defective structures such as chemically reduced graphene oxide were studied with respect to their use in electrodes and sensors. Their electrochemical properties and utility in terms of fabrication of sensing devices are compared. Specifically, the electrodes have been applied to reductive amperometric determination of hydrogen peroxide. Low-defect graphene (SG) was obtained through mechanical exfoliation of natural graphite, while higher-defect graphenes were produced by chemical vapor deposition (CVDG) and by chemical oxidation of graphite and subsequent reduction (rGO). The carbonaceous materials were mainly characterized by Raman microscopy. They were applied as electrode material and the electrochemical behavior was investigated by chronocoulometry, cyclic voltammetry, electrochemical impedance spectroscopy and amperometry and compared to a carbon disc electrode. It is shown that the quality of the graphene has an enormous impact on the amperometric performance. The use of carbon materials with many defects (like
\end{abstract}

Alexander Zöpfl and Masoumeh Sisakthi contributed equally to this work.

Electronic supplementary material The online version of this article (doi:10.1007/s00604-015-1600-y) contains supplementary material, which is available to authorized users.

Thomas Hirsch

thomas.hirsch@ur.de

1 Institute of Analytical Chemistry, Chemo- and Biosensors, University of Regensburg, Universitaets-Strasse 31, 93053 Regensburg, Germany

2 Institute of Experimental and Applied Physics, Micro- and Nanophysics, University of Regensburg, Universitaets-Strasse 31, 93053 Regensburg, Germany
rGO) does not result in a significant improvement in signal compared to a plain carbon disc electrode. The sensitivity is $173 \mathrm{~mA} \cdot \mathrm{M}^{-1} \cdot \mathrm{cm}^{-2}$ in case of using CVDG which is about 50 times better than that of a plain carbon disc electrode and about 7 times better than that of rGO. The limit of detection for hydrogen peroxide is $15.1 \mu \mathrm{M}$ (at a working potential of $-0.3 \mathrm{~V}$ vs SCE) for CVDG. It is concluded that the application of two-dimensional carbon nanomaterials offers large perspectives in amperometric detection systems due to electrocatalytic effects that result in highly sensitive detection.

Keywords Graphene $\cdot$ Reduced graphene oxide $\cdot$ Hydrogen peroxide $\cdot$ Amperometry $\cdot$ Electrical impedance spectroscopy $\cdot$ Chronocoulometry $\cdot$ Cyclic voltammetry $\cdot$ Raman spectroscopy

\section{Introduction}

Graphene, a carbon nanomaterial comprising a single $\mathrm{sp}^{2}$ carbon atomic layer, has attracted attention since its first exfoliation in 2004 [1, 2]. The material is known for excellent electrical properties like large redox active surface area (2, $630 \mathrm{~m}^{2} \mathrm{~g}^{-1}$ ) [3] and high electron mobility (up to $\mu \approx 250$, $000 \mathrm{~cm}^{2} \cdot \mathrm{V}^{-1} \cdot \mathrm{s}^{-1}$ at room temperature) [4], both making this material attractive to be used as electrode in electrochemical applications. It has also been reported that carbon nanomaterials comprise electrocatalytic effects in amperometric detection systems [5]. Therefore numerous studies deal with graphene as electrode material in (bio) sensing applications [6], but often these materials are not exactly defined in their chemical structure, shape, size, or number of layers [7]. Even within the same production technique a poorly defined material will be received, often with slightly different properties from batch to batch. 
To date, most graphene employed in electrochemical analysis is chemically synthesized via oxidative methods with subsequent reduction, introducing a lot of defects and heteroatoms $[8,9]$. These materials incorporate oxygen containing groups which strongly influences the electrochemical properties. Graphene derived from other methods like chemical vapor deposition (CVD) $[10,11]$ also contains structural defects or impurities generated by its transfer from a metallic support to an insulating substrate. Many other methods have been developed so far which allow the preparation of graphene of various sizes, shapes and quality [12]. The methods most commonly used can be classified by mechanical [1] or chemical exfoliation [13]. All of these methods are advantageous in some ways, and except the mechanical exfoliation they allow to produce large quantities of graphene. The defects in the carbon nanomaterials do not only negatively influence the conductance [14] but also offer the possibility of functionalization with biomolecules and/or metal and metal oxide nanoparticles. This step is mandatory to introduce selectivity to the system with the perspective of creating sensor platforms in great variability. Tailoring the size and morphology of the graphene in combination with other nanomaterials, results in composite materials with enhanced sensing performance [15].

There are many reports on amperometric detection systems for $\mathrm{H}_{2} \mathrm{O}_{2}$ based on graphene materials. An enzymatic system using horseradish peroxidase immobilized onto graphenebased electrodes led to a limit of detection of $0.1 \mu \mathrm{M}$ [16] Up to now, there are still drawbacks assigned to this approach like complex immobilization protocols, low temporal stability, and massive influence of $\mathrm{pH}$, temperature, or humidity on the enzyme activity. Great efforts have been made to develop nonenzymatic peroxide detection systems, using noble metals [17, 18], metal oxides and sulfides [19], and composite materials with other carbon nanomaterials [20]. These modifications have been chosen for its enhanced electron transfer rates and for catalytic activity, resulting in higher sensitivity. Lin et al. recently presented a electrochemical detection of $\mathrm{H}_{2} \mathrm{O}_{2}$ based on a carbon nanotube $\mathrm{MoS}_{2}$ composite, with an outstanding limit of detection of $5 \mathrm{nM}$ [21]. Nevertheless, the role of the individual materials within these composites is not fully understood yet.

Therefore we investigate the influence of the choice of the graphene material itself on the amperometric properties in direct hydrogen peroxide detection. The graphene materials in this study were prepared by mechanical exfoliation (single layer graphene, SG), chemical vapor deposition (chemical vapor deposited graphene, CVDG) and chemical exfoliation (reduced graphene oxide, rGO), comprising a different degree of defects. All types of carbon materials have been characterized with Raman spectroscopy, cyclic voltammetry, electrochemical impedance spectroscopy and amperometry. The sensitivity in direct detection of hydrogen peroxide has been investigated. From these results the influence of the defects in the carbon nanomaterial can be assigned to the sensitivity which can be achieved.

\section{Experimental}

\section{Materials and instrumentations}

Unless otherwise stated, all chemicals were of analytical grade and purchased from Merck (www.merck.de, Darmstadt, Germany) or Sigma-Aldrich (www.sigma-aldrich.de, Steinheim, Germany) and used without further purification. Ultra-pure water $\left(0.055 \mu \mathrm{S} \cdot \mathrm{cm}^{-1}\right)$ was used in all experiments.

Raman spectra were recorded by a Thermo Scientific DXR Raman microscope with a $532 \mathrm{~nm}$ excitation laser, a laser power of $10 \mathrm{~mW}$, and a spot size of $2.1 \mu \mathrm{m}$ in diameter. All spectra have been measured 10 times with an integration of $1 \mathrm{~s}$. Electrochemical characterizations and measurements were performed on a CH Instrument electrochemical analyzer Model 602a. The three-electrode system consisted of a Pt wire as counter electrode and a saturated calomel electrode (SCE) as reference electrode. A custom build carbon disc electrode, comprising a carbon fibre composite (www.conrad.de, Regensburg, Germany) with $2 \mathrm{~mm}$ diameter implemented in Teflon or the differently prepared graphene materials (SG, CVDG, and rGO) assembled on a silicon wafer substrate electrically contacted by gold leads were used as working electrode.

\section{Preparation of different graphene materials}

Single layer graphene (SG) was obtained through micromechanical exfoliation of graphite flakes (NGS Naturgraphit GmbH, Leinburg, Germany) using an adhesive tape, and transferred onto a Si substrate with a $300 \mathrm{~nm}$ thick $\mathrm{SiO}_{2}$ insulating layer. This kind of graphene flakes is random in shape and size, with a typical size of about $50 \mu \mathrm{m}^{2}$.

Graphene derived by CVD (CVDG) was purchased on $\mathrm{Si} /$ $\mathrm{SiO}_{2}$ substrates (www.graphenea.com, San Sebastian, Spain). Oxygen plasma etching was used to remove excess graphene, forming graphene electrodes of about $0.04 \mathrm{~mm}^{2}$ in size.

Reduced graphene oxide (rGO) was prepared by a slightly modified Hummers method [8] with subsequent reduction [22], which has been described in a previous work [23]. Briefly, $1 \mathrm{~g}$ of China flake graphite $(\mathrm{K}$. W. Thielmann \& Cie KG, Grolsheim, Germany) was mixed with $0.75 \mathrm{~g} \mathrm{NaNO}_{3}$ in $75 \mathrm{~mL}$ conc. $\mathrm{H}_{2} \mathrm{SO}_{4}$ and $4.5 \mathrm{~g} \mathrm{KMnO}_{4}$ was added in small portions under vigorous stirring and cooling in an ice bath. The mixture was sonicated for $3 \mathrm{~h}$ and stirred for 3 days at room temperature. After the addition of $75 \mathrm{~mL}$ of $5 \% \mathrm{H}_{2} \mathrm{SO}_{4}$ the mixture was heated at $100{ }^{\circ} \mathrm{C}$ for $2 \mathrm{~h}$, followed by an 
addition of $15 \mathrm{~mL}$ of $30 \% \mathrm{H}_{2} \mathrm{O}_{2}$ and constant stirring for $1 \mathrm{~h}$ at room temperature. For purification, the product was washed with the following solutions: four times in $3 \% \mathrm{H}_{2} \mathrm{SO}_{4}$ containing $0.5 \% \mathrm{H}_{2} \mathrm{O}_{2}$; two times in $3 \% \mathrm{HCl}$; three times in water. Finally it was dialyzed against $2 \mathrm{~L}$ water $(14 \mathrm{kDa}$ cutoff) for 10 days, changing the water three times. For the chemical reduction of GO, $7 \mathrm{~mL}$ of a GO suspension $\left(0.5 \mathrm{~g} \cdot \mathrm{L}^{-1}\right)$ were mixed with $31 \mu \mathrm{L}$ of $32 \% \mathrm{NH}_{3}$. After adding $5 \mu \mathrm{L}$ of $98 \%$ hydrazine hydrate the reaction mixture was refluxed for $1 \mathrm{~h}$ at $100{ }^{\circ} \mathrm{C}$. The resulting black suspension was washed with water and isolated by centrifugation.

\section{Preparation of graphene modified electrodes}

CVD-grown graphene was cut to a rectangular area employing electron beam lithography and reactive ion etching $\left(\mathrm{O}_{2} / \mathrm{Ar}\right)$. To provide an electrical contact, the graphene was partially metallized through evaporation of $\mathrm{Ti} / \mathrm{Au}(5 \mathrm{~nm} / 60 \mathrm{~nm})$, followed by an acetone/ipropanol lift-off process. Electrodes consisting of rGO were produced via drop casting of $1 \mu \mathrm{L}$ of an aqueous $\mathrm{rGO}$ suspension $\left(0.25 \mathrm{~g} \cdot \mathrm{L}^{-1}\right)$ on microelectrodes with interdigital structure of thin gold layers and dried at room temperature. A final annealing step of $230{ }^{\circ} \mathrm{C}$ for $30 \mathrm{~s}$ was applied afterwards. All gold areas in contact with the electrolyte were shielded by dipping the electrode into a solution of $100 \mu \mathrm{M} 1$-mercaptooctadecane in ethanol, to form a self assembled monolayer. The successful shielding of the gold contacts exposed to the electrolyte was proven by cyclic voltammetry (Figure S1). The different types of graphene modified electrodes on silicon substrates are depicted in Fig. 1.

\section{Electrochemical characterization and measurements}

The modified electrodes and the carbon disc electrode were characterized with cyclic voltammetry, chronocoulometry, electrochemical impedance spectroscopy (EIS) and amperometry. For electrochemical experiments, the supporting electrolytes were $0.1 \mathrm{M} \mathrm{KCl}$ or $10 \mathrm{mM}$ phosphate buffer with $140 \mathrm{mM} \mathrm{NaCl}$ (pH 7.4). Before each measurement Argon was bubbled through the solutions to remove dissolved oxygen. All experiments were performed at room temperature. The amperometric response towards $\mathrm{H}_{2} \mathrm{O}_{2}$ was investigated in a continuously stirred electrolyte solution (10 mM phosphate buffer, $140 \mathrm{mM} \mathrm{NaCl}, \mathrm{pH}$ 7.4) by doubling the concentration of peroxide after each injection. The studied analyte concentration covers a range from 25 to $25.6 \mathrm{mM}$.

\section{Results and discussion}

Raman spectroscopy is established as a versatile characterization method for 2D carbon nanomaterials. Information regarding the number of layers, the amount of defects and impurities can be obtained. Therefore all graphene modified electrodes have been investigated by Raman microscopy. As can be seen in Fig. 2, no observable D peak, which indicates defects in the aromatic system of the graphene, is evident in the spectrum of SG, which proves the high structural order for this type of graphene. For CVDG a small D peak is observed at $1342 \mathrm{~cm}^{-1}$ (Figure S2B) and especially for rGO the D band at $1352 \mathrm{~cm}^{-1}$ becomes dominant and broader, indicating a high level of disorder [25]. A symmetric single 2D peak can be found in the spectra of SG and CVDG at 2676 and $2684 \mathrm{~cm}^{-1}$, respectively. Further, the significant higher intensity compared to the $\mathrm{G}$ band at 1583 and $1590 \mathrm{~cm}^{-1}$ indicate that these materials consist of high quality single layer graphene [26]. In rGO, the noticeable change in shape and intensities of the D, G, and 2D band illustrates the significant presence of multiple layers, lattice disorder and inhomogeneity in this type of graphene. Applying an empirical TuinstraKoenig relation [27] which refers to the crystallite size of graphite, the diameter of the $\mathrm{sp}^{2}$ regions in $\mathrm{rGO}$ was calculated to approximately $19 \mathrm{~nm}$.

\section{Electrochemical characterization of different graphene electrode materials}

Due to the different nature of the various types of graphene one cannot prepare electrodes of the same size. But size has huge influence on the sensitivity of the modified electrodes. The geometric area gives only a rough estimation, therefore chronocoulometric measurements in the presence of $0.1 \mathrm{mM} \mathrm{K}_{3}\left[\mathrm{Fe}(\mathrm{CN})_{6}\right]$ were performed in order to estimate
Fig. 1 Microscopic pictures of electrodes prepared with different graphene materials: a SG, b CVDG and $\mathbf{c}$ rGO. In (A) and (B) the graphene can be visualized by the microscope due to the interference between reflection paths of the air- $\mathrm{SiO}_{2}$ and $\mathrm{SiO}_{2}$-Si interface [24]
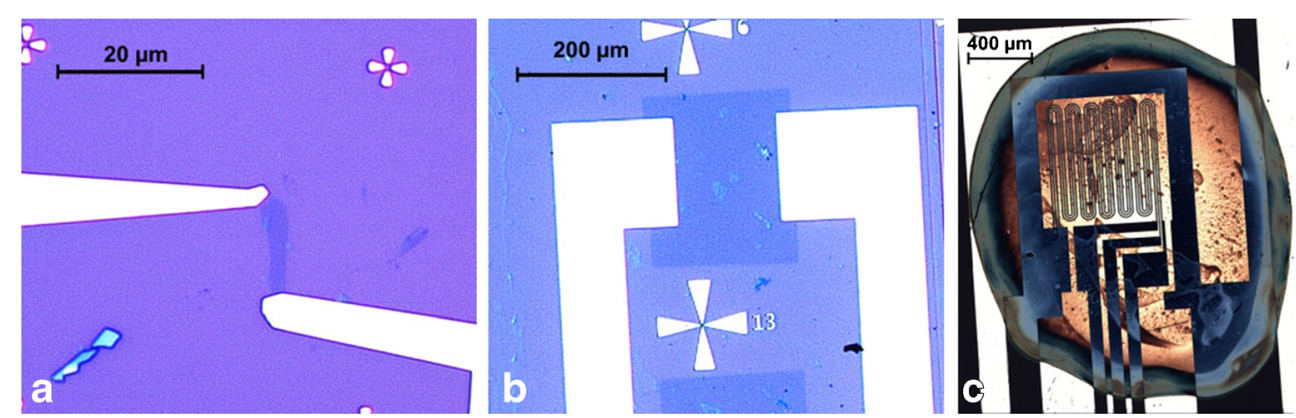


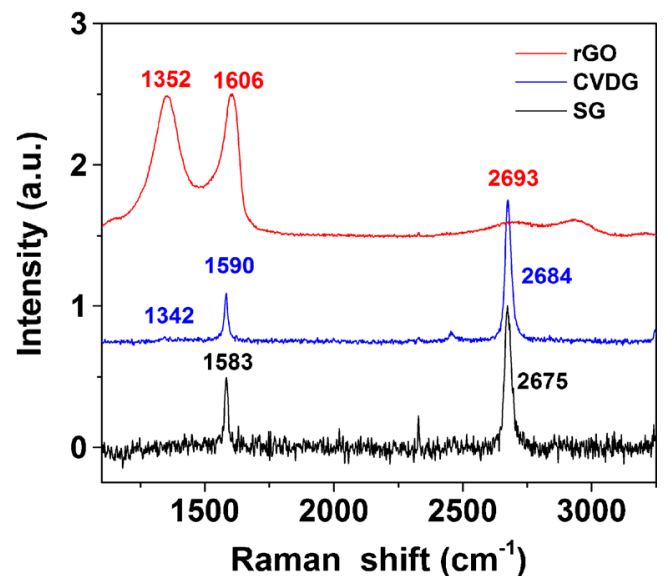

Fig. 2 Raman spectra of SG-, CVDG- and rGO-modified electrodes

the total electroactive area of each electrode. The effective surface area of the carbon disc electrode was calculated to be $2.9 \cdot 10^{6}, 2.6 \cdot 10^{6} \mu^{2}$ for $\mathrm{rGO}, 3.9 \cdot 10^{4} \mu \mathrm{m}^{2}$, for CVDG, and $51 \mu \mathrm{m}^{2}$ for the SG electrode, respectively. These values were further used for the calculation of current densities.

Electrochemical impedance spectroscopy provides information of the interfacial properties of the electrodes. In Fig. 3 the Nyquist and Bode plots of the three types of graphene modified electrodes and the carbon disc electrode in presence of $5 \mathrm{mM} \mathrm{K}_{4}\left[\mathrm{Fe}(\mathrm{CN})_{6}\right]$ containing $0.1 \mathrm{M} \mathrm{KCl}$ at $0.2 \mathrm{~V}$ vs SCE are shown. Electrodes modified with SG, CVDG and rGO cannot be fully described by a Randles equivalent circuit, consisting of the electrolyte resistance $\mathrm{R}_{\mathrm{e}}$ in series with a parallel combination of double-layer capacitance $\left(\mathrm{C}_{\mathrm{dl}}\right)$, an impedance representing the charge transfer resistance $\left(\mathrm{R}_{\mathrm{ct}}\right)$ and the Warburg element $\left(\mathrm{Z}_{\mathrm{w}}\right)$ taking diffusion into account. The results from the electrochemical impedance spectroscopy do not take into account that there is a huge difference in the electrode surface area as can be seen from the results of the chronocoulometric measurements. For high frequencies the impedance $(Z)$ for all systems is approaching the electrolyte resistance. For small frequencies the chargetransfer resistance increases for the different electrodes in the order carbon disc, rGO, CVDG to SG. The behavior of the
CVDG can be explained by impurities adsorbed on the graphene. These can be a consequence of the transfer process [10]. Here, a polymer layer will is deposited on top of the graphene layer; the metallic substrate where the graphene was deposited is etched away so that the polymer modified graphene flake swims on top of the solution. After transfer to the silicon wafer the polymer on top of the graphene is washed away by organic solvents. By this process it is likely that polymer residues can remain. The Nyquist plot in Fig. 3a suggests that additional impedance for adsorbates need to be applied to the equivalent circuit. For the rGO system, which consists of multilayers and comprises many defects, it is expected that the impedance spectrum is not in accordance to the simple model described by the Randles equivalent circuit. The SG modified electrode as well as the carbon disc electrode show a diffusion process for the redox species at low frequencies. The higher double-layer capacitance of SG modified electrodes in contrast to the carbon disc electrode as can be seen from the imaginary part in the Nyquist plot (Z") can be attributed to the hydrophobic character of the aromatic system which is repelling the redox species. After normalization of the total impedance $\mathrm{Z}$ to the electroactive surface area the charge transfer resistance of carbon disc electrode is approximately $1.2 \cdot 10^{6}$ times higher than for the SG modified electrode, for CVDG $\mathrm{R}_{\mathrm{ct}}$ per area is $1.7 \cdot 10^{3}$ times, for rGO 1.3 . $10^{5}$ times higher. This shows that all graphene types enhance the electron transfer of the ferro/ferricyanide redox system compared to a plain carbon disc electrode. The differences between the three different types are in accordance to the increase of the number of defects of the two-dimensional carbon nanomaterial. The numbers given here are only rough estimations not taking into account the influence of the border of the CVDG and SG. A detailed investigation of different flake sizes will complete this picture.

\section{Direct amperometric detection of hydrogen peroxide}

The reduction of $\mathrm{H}_{2} \mathrm{O}_{2}$ on the various graphene electrodes was studied by cyclic voltammetry (Fig. 4) in the presence of
Fig. 3 Nyquist plot (a) and Bode plot (b) derived from electrochemical impedance spectroscopy in $0.1 \mathrm{M} \mathrm{KCl}$ and $5 \mathrm{mM} \mathrm{K}_{4}\left[\mathrm{Fe}(\mathrm{CN})_{6}\right]$ for carbon disc, rGO, CVDG and SG modified electrodes (at $0.2 \mathrm{~V}$ vs SCE with an amplitude of $5 \mathrm{mV}$ )
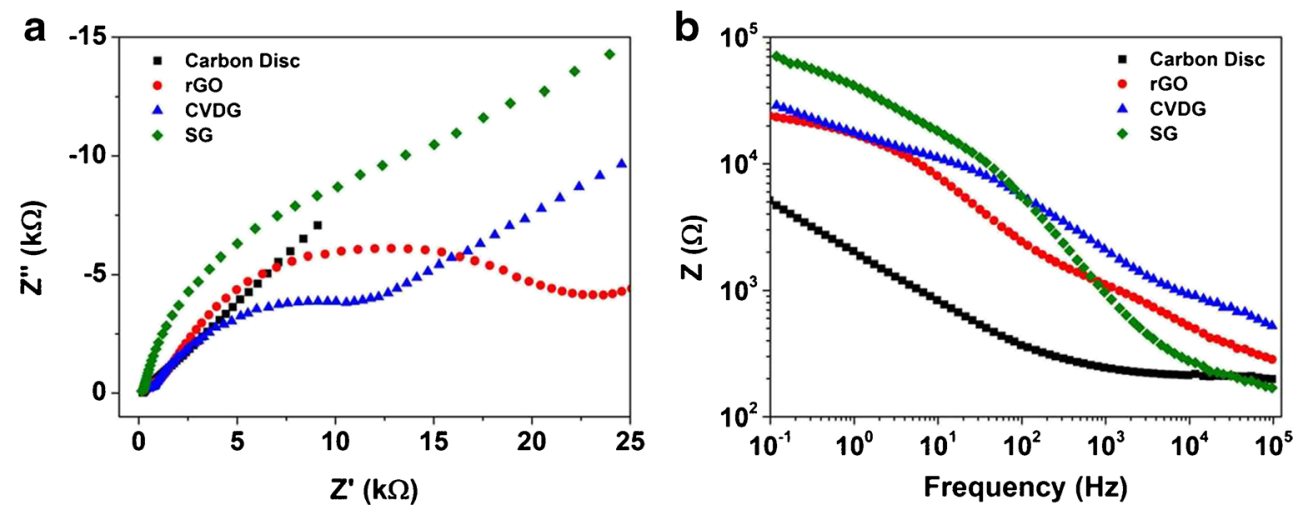
Fig. 4 Steady state current density/voltage cycles of a rGO, $\mathbf{b}$ CVDG, $\mathbf{c}$ SG, and $\mathbf{d}$ carbon disc electrode in phosphate buffered saline $\mathrm{pH} 7.4$ and after addition of $10 \mathrm{mM} \mathrm{H}_{2} \mathrm{O}_{2}$ vs SCE (scan rate $0.1 \mathrm{~V} \mathrm{~s}^{-1}$ )
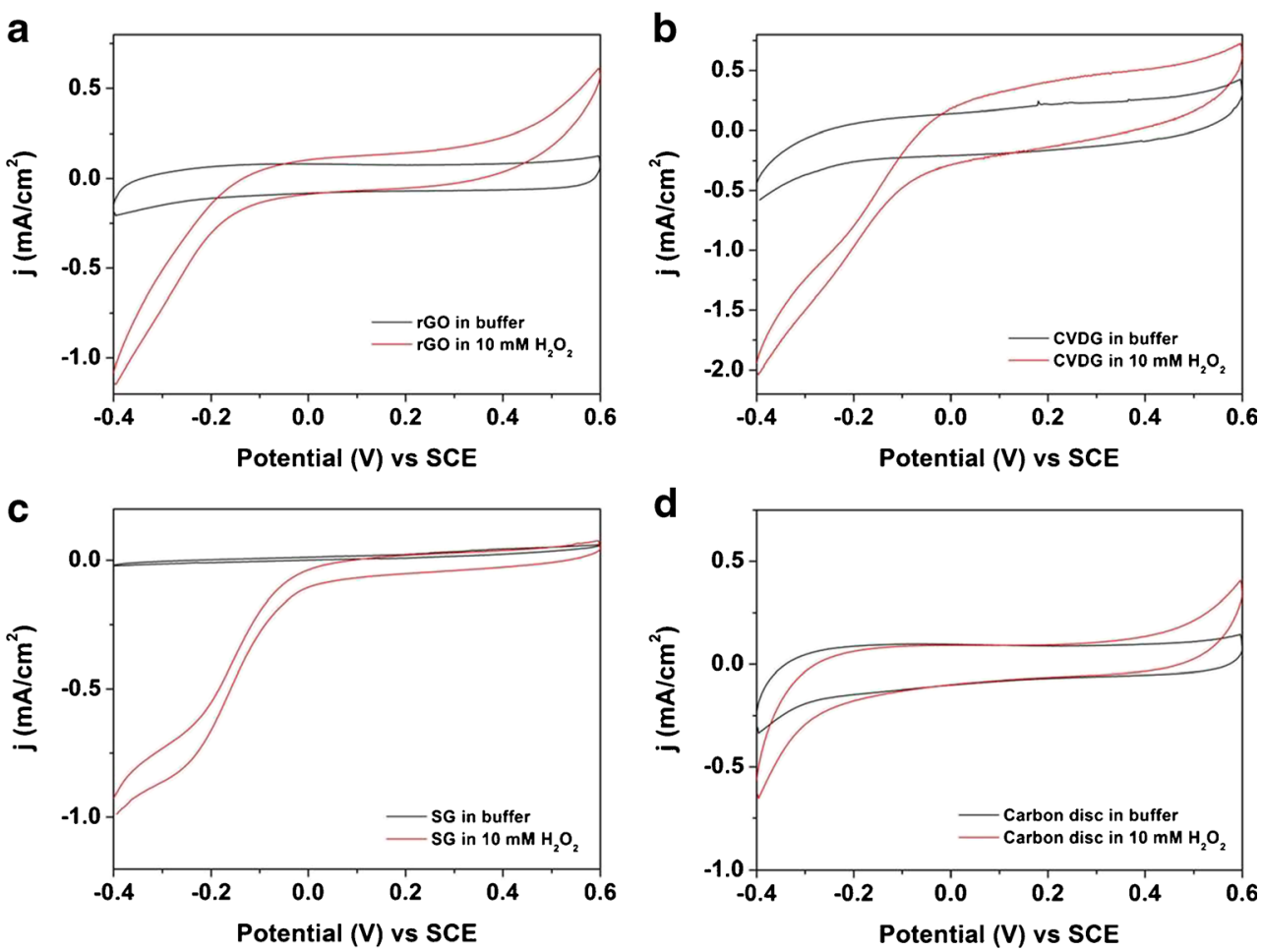

$10 \mathrm{mM} \mathrm{H} \mathrm{O}_{2}$ in phosphate buffered saline at $\mathrm{pH}$ 7.4. Comparison of the cyclic voltammograms before and after applying $10 \mathrm{mM} \mathrm{H}_{2} \mathrm{O}_{2}$ shows that after the addition of $\mathrm{H}_{2} \mathrm{O}_{2}$, the cathodic current increased. The increase for electrodes modified with CVDG and SG can be already observed at low potentials of $-0.1 \mathrm{~V}$ vs SCE. This is at lower potentials than for $\mathrm{rGO}$ and carbon disc electrode and can be attributed to an electrocatalytic effect of the carbon nanomaterials.

The change in the current density under exactly the same conditions is significantly higher for the graphene materials than for the carbon disc electrode (Fig. 5). At a working potential of $-0.3 \mathrm{~V}$ vs SCE the current flow is enhanced about 4 times for rGO, 5 times for SG and 8 times for CVDG. Especially CVDG electrodes provide maximum response, maybe due to the best ratio of pristine graphene lattice to defects and edges.

In order to get high selectivity in real sample analysis it is desired to determine the analytes at potentials close to $0 \mathrm{~V}$. We found that at very low potentials graphene with low number of defects, here CVDG and SG, show nearly the same enhancement in the signal whereas $\mathrm{rGO}$ as very defective material shows a behavior more like the carbon disc electrode (Fig. 5). For more negative electrochemical potentials all four types of carbon materials give an individual signal enhancement. From this result one can conclude that for working potentials lower than $-0.1 \mathrm{~V}$ vs SCE it is mandatory to use high quality graphene with low number of defects in order to get any benefit in using a two dimensional carbon nanomaterial as electrode in amperometric detection of $\mathrm{H}_{2} \mathrm{O}_{2}$.
All three types of graphene-modified electrodes have been tested in an amperometric setup to detect peroxide (Fig. 6 and S3A). A constant potential of $-0.3 \mathrm{~V}$ vs SCE was applied. From Figures $\mathrm{S} 3 \mathrm{~B} / \mathrm{C}$ it can be observed that the reductive current response of all electrodes increase with increasing $\mathrm{H}_{2} \mathrm{O}_{2}$ concentration. For low concentrations of the analyte one can see that low-defect graphene (SG) does not exhibit notable changes in the current. One reason is that the size of these flakes is very small and therefore the total signal is very small. After normalization of all signals to the total electroactive surface area, the situation changed (Figure S3D): the sensitivity of SG for low amounts of $\mathrm{H}_{2} \mathrm{O}_{2}$ can be seen. An increase of absolute sensitivity is observed, starting from carbon disc $\left(3.2 \mathrm{~mA} \cdot \mathrm{M}^{-1} \cdot \mathrm{cm}^{-2}\right)$ to $\mathrm{rGO}$

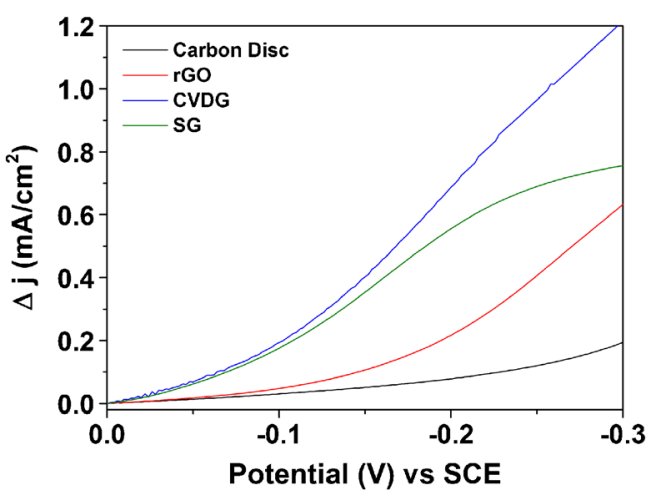

Fig. 5 Change in current density for $\mathrm{H}_{2} \mathrm{O}_{2}$ reduction during the cyclic voltammetry in phosphate buffered saline $\mathrm{pH} 7.4$ with $10 \mathrm{mM} \mathrm{H}_{2} \mathrm{O}_{2}$ vs SCE (scan rate $0.1 \mathrm{~V} \mathrm{~s}^{-1}$ ) 


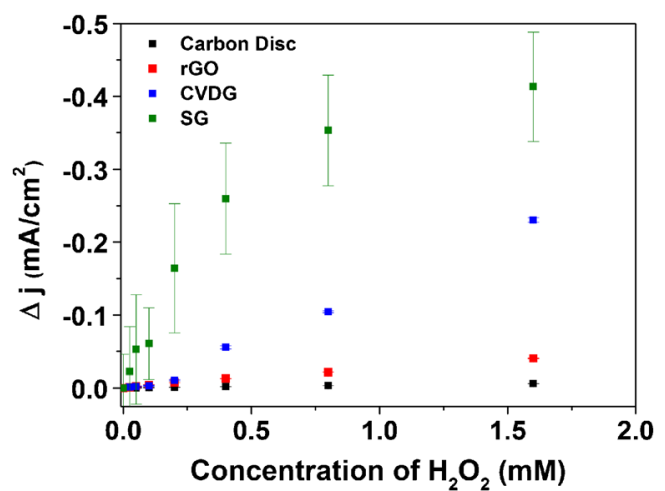

Fig. 6 Concentration dependency of the amperometric response at $-0.3 \mathrm{~V}$ vs SCE upon successive addition of $0.025-25.6 \mathrm{mM} \mathrm{H}_{2} \mathrm{O}_{2}$ into continuously stirred phosphate buffered saline $\mathrm{pH}$ 7.4. $(n=3)$

$\left(25 \mathrm{~mA} \cdot \mathrm{M}^{-1} \cdot \mathrm{cm}^{-2}\right)$ to $\mathrm{CVDG}\left(173 \mathrm{~mA} \cdot \mathrm{M}^{-1} \cdot \mathrm{cm}^{-2}\right)$, and SG modified electrodes $\left(202 \mathrm{~mA} \cdot \mathrm{M}^{-1} \cdot \mathrm{cm}^{-2}\right)$. Again, a lower number of defects lead to a higher sensitivity towards $\mathrm{H}_{2} \mathrm{O}_{2}$. Nevertheless, the noise level of SG electrodes is higher than the other electrodes which can be attributed to the low absolute current values. Noise at an amperometric electrode is closely associated with $\mathrm{C}_{\mathrm{d} 1}$ and the quality of attachment to the support. Calculating the limit of detection from a signal-tonoise ratio of three, values of $9.2 \mu \mathrm{M}$ for $\mathrm{rGO}, 15.1 \mu \mathrm{M}$ for CVDG and $651.5 \mu \mathrm{M}$ for SG electrodes are obtained.

These findings indicate the great perspective of graphene in amperometric detection systems. For comparison, the $\mathrm{H}_{2} \mathrm{O}_{2}$ sensing performance of different graphene based electrode materials from previous reports are displayed in Table 1. All these works are based on chemically derived graphene, which has been further modified with metal or metal oxide nanomaterials to improve sensitivity. One can clearly see that hybrid nanomaterials lead to better sensitivity. From our study one can suggest that there could be further improvement by using graphene of low number of defects. For taking benefit of the electrocatalytic effect of graphene as well as from the signal enhancement it is mandatory to use electrodes consisting of high quality graphene, which means, to use a 2D nanomaterial with as low number of defects and impurities as possible. Up to now it is challenging to provide such a high quality graphene on an insulating material with electric contacts in reasonable quantities and of adequate size for developing sensor applications operated with a low cost potentiostat. By taking a look to the relative signal changes obtained with the different types of electrodes used in this study one can see that SG performs best, but accompanied by huge error bars due to an enhancement of the noise. This could be overcome by using bigger graphene flakes or by better potentiostats. Good signal enhancement can also be obtained with CVDG. Nevertheless the fabrication of one of such electrodes is a time consuming complex process. This material is already commercially available. But the drawback in using this material for an application is that there is a lack of a technique for a clean and easy transfer of this material onto microelectrodes providing a good electrical contact. This material offers the possibility to be functionalized chemically [33] or by plasma treatment [34]. It is expected that with this technique selectivity can be introduced and functionalized graphene for amperometric detection systems will be designed. The rGO does not result in such great improvement in contrast to the carbon disc electrode, but here processing of the material as well as functionalization is easy. Therefore it offers a pathway to interesting composite materials for electrochemical sensors.

\section{Conclusion}

We have studied non-enzymatic detection of hydrogen peroxide using electrodes based on various types of graphene, without utilizing any functionalization or modification steps. The graphene materials studied include SG, CVDG and rGO. The
Table 1 Comparison of analytical performance of various graphene and graphene composite materials used for the electrochemical detection of $\mathrm{H}_{2} \mathrm{O}_{2}$

\begin{tabular}{lllll}
\hline Electrode material & Potential [V] & Sensitivity $\left[\mathrm{mA} \cdot \mathrm{M}^{-1} \cdot \mathrm{cm}^{-2}\right]$ & LOD $[\mu \mathrm{M}]$ & Reference \\
\hline PB/Graphene & $-0.05(\mathrm{vs} \mathrm{Pt})$ & 196.6 & 1.9 & {$[28]$} \\
$\mathrm{CoOxNPs} / \mathrm{ERG}$ & $0.75(\mathrm{vs} \mathrm{SCE})$ & 148.6 & 0.2 & {$[19]$} \\
$\mathrm{MnO}_{2} / \mathrm{GO}$ & $-0.3(\mathrm{vs} \mathrm{SCE})$ & 8.2 & 0.8 & {$[29]$} \\
$\mathrm{AgNPs} / \mathrm{PQ} 11 /$ Graphene & $-0.4(\mathrm{vs} \mathrm{Ag} / \mathrm{AgCl})$ & 56.6 & 28 & {$[30]$} \\
Polydopamine-rGO/Ag & $-0.5(\mathrm{vs} \mathrm{Ag} / \mathrm{AgCl})$ & 355.8 & 2.1 & {$[31]$} \\
rGO-PMS/AuNPs & $-0.75(\mathrm{vs} \mathrm{Ag} / \mathrm{AgCl})$ & 39.2 & 0.06 & {$[32]$} \\
rGO & $-0.3(\mathrm{vs} \mathrm{SCE})$ & 25 & 9.2 & This Work \\
$\mathrm{CVDG}$ & $-0.3(\mathrm{vs} \mathrm{SCE})$ & 173 & 15.1 & This Work \\
$\mathrm{SG}$ & $-0.3(\mathrm{vs} \mathrm{SCE})$ & 202 & 651.5 & This Work \\
\hline
\end{tabular}

$P B$ Prussian Blue, CoOxNPs Cobalt oxide nanoparticles, ERGO Electrochemically reduced graphene oxide, AgNPs Silver nanoparticles, $P Q 11$ Poly[(2-ethyldimethylammonioethyl methacrylate ethyl sulfate)-co-(1vinylpyrrolidone)], PMS Periodic mesopourous silica, $\mathrm{AuNPs}$ Gold nanoparticles, $\mathrm{Ag} / \mathrm{AgCl}$ Saturated silver/ silver chloride reference electrode 
effects of the device fabrication and sensing performance were investigated. Results reveal that all three graphene materials exhibit sensitivity to the catalytic reduction of $\mathrm{H}_{2} \mathrm{O}_{2}$ and are able to detect $\mathrm{H}_{2} \mathrm{O}_{2}$ concentrations below $0.1 \mathrm{mM}$, showing fast amperometric response upon successive additions of $\mathrm{H}_{2} \mathrm{O}_{2}$. Moreover, it is clearly demonstrated that mechanically exfoliated graphene as well as graphene prepared by CVD are promising candidates for sensor applications, due to their efficiency in better detection of hydrogen peroxide with higher sensitivity compared to rGO. However, due to the laborious process needed for production of exfoliated graphene and its irregularity in shape, size and location, this type of graphene is more suitable for theoretical study and proof-of-concept demonstration. In comparison, the CVDG suits commercial purposes and mass production, since it offers uniform continuous films at particular locations and with desired geometries, providing a superior candidate for fabrication of high sensitivity biosensors and sensor arrays. Such carbon nanomaterials can also be used as biosensors by further functionalization with enzymes.

Acknowledgments This work was supported by DFG Research Training Electronic Properties of Carbon Based Nanostructures (GRK 1570).

\section{References}

1. Novoselov KS, Geim AK, Morozov SV, Jiang D, Zhang Y, Dubonos SV, Grigorieva IV, Firsov AA (2004) Electric field effect in atomically thin carbon films. Science 306:666-669. doi:10.1126/ science. 1102896

2. Ferrari AC, Bonaccorso F, Fal'ko V, Novoselov KS, Roche S, Bøggild P, Borini S, Koppens FHL, Palermo V, Pugno N, Garrido JA, Sordan R, Bianco A, Ballerini L, Prato M, Lidorikis E, Kivioja J, Marinelli C, Ryhänen T, Morpurgo A, Coleman JN, Nicolosi V, Colombo L, Fert A, Garcia-Hernandez M, Bachtold A, Schneider GF, Guinea F, Dekker C, Barbone M, Sun Z, Galiotis C, Grigorenko AN, Konstantatos G, Kis A, Katsnelson M, Vandersypen L, Loiseau A, Morandi V, Neumaier D, Treossi E, Pellegrini V, Polini M, Tredicucci A, Williams GM, Hong BH, Ahn J-H, Kim JM, Zirath H, van Wees BJ, van der Zant H, Occhipinti L, Matteo AD, Kinloch IA, Seyller T, Quesnel E, Feng X, Teo K, Rupesinghe N, Hakonen P, Neil SRT, Tannock Q, Löfwander T, Kinaret J (2015) Science and technology roadmap for graphene, related two-dimensional crystals, and hybrid systems. Nanoscale 7:4598-4810. doi:10.1039/C4NR01600A

3. Stoller MD, Park S, Zhu Y, An J, Ruoff RS (2008) Graphene-based ultracapacitors. Nano Lett 8:3498-3502. doi:10.1021/n1802558y

4. Geim AK (2009) Graphene: status and prospects. Science 324: 1530-1534. doi:10.1126/science. 1158877

5. Pérez-López B, Merkoçi A (2012) Carbon nanotubes and graphene in analytical sciences. Microchim Acta 179:1-16. doi:10.1007/ s00604-012-0871-9

6. Zhou M, Zhai Y, Dong S (2009) Electrochemical sensing and biosensing platform based on chemically reduced graphene oxide. Anal Chem 81:5603-5613. doi:10.1021/ac900136z

7. Kochmann S, Hirsch T, Wolfbeis OS (2012) Graphenes in chemical sensors and biosensors. TrAC Trends Anal Chem 39:87-113. doi: $10.1016 / j$.trac.2012.06.004
8. Hummers WS, Offeman RE (1958) Preparation of graphitic oxide. J Am Chem Soc 80:1339-1339. doi:10.1021/ja01539a017

9. Marcano DC, Kosynkin DV, Berlin JM, Sinitskii A, Sun Z, Slesarev A, Alemany LB, Lu W, Tour JM (2010) Improved synthesis of graphene oxide. ACS Nano 4:4806-4814. doi:10.1021/ nn1006368

10. Kim KS, Zhao Y, Jang H, Lee SY, Kim JM, Kim KS, Ahn J-H, Kim P, Choi J-Y, Hong BH (2009) Large-scale pattern growth of graphene films for stretchable transparent electrodes. Nature 457: 706-710. doi:10.1038/nature07719

11. Shang NG, Papakonstantinou P, McMullan M, Chu M, Stamboulis A, Potenza A, Dhesi SS, Marchetto H (2008) Catalyst-free efficient growth, orientation and biosensing properties of multilayer graphene nanoflake films with sharp edge planes. Adv Funct Mater 18:3506-3514. doi:10.1002/adfm.200800951

12. Novoselov KS, Fal'ko VI, Colombo L, Gellert PR, Schwab MG, Kim K (2012) A roadmap for graphene. Nature 490:192-200. doi: 10.1038 /nature 11458

13. Park S, Ruoff RS (2009) Chemical methods for the production of graphenes. Nat Nanotechnol 4:217-224. doi:10.1038/nnano.2009.58

14. Tang L, Wang Y, Li Y, Feng H, Lu J, Li J (2009) Preparation, structure, and electrochemical properties of reduced graphene sheet films. Adv Funct Mater 19:2782-2789. doi:10.1002/adfm. 200900377

15. Ambrosi A, Chua CK, Bonanni A, Pumera M (2014) Electrochemistry of graphene and related materials. Chem Rev 114:7150-7188. doi:10.1021/cr500023c

16. Lu L-M, Qiu X-L, Zhang X-B, Shen G-L, Tan W, Yu R-Q (2013) Supramolecular assembly of enzyme on functionalized graphene for electrochemical biosensing. Biosens Bioelectron 45:102-107. doi:10.1016/j.bios.2013.01.065

17. Chen S, Yuan R, Chai Y, Hu F (2012) Electrochemical sensing of hydrogen peroxide using metal nanoparticles: a review. Microchim Acta 180:15-32. doi:10.1007/s00604-012-0904-4

18. Cui X, Wu S, Li Y, Wan G (2014) Sensing hydrogen peroxide using a glassy carbon electrode modified with in-situ electrodeposited platinum-gold bimetallic nanoclusters on a graphene surface. Microchim Acta 182:265-272. doi:10.1007/s00604-014-1321-7

19. Li S-J, Du J-M, Zhang J-P, Zhang M-J, Chen J (2014) A glassy carbon electrode modified with a film composed of cobalt oxide nanoparticles and graphene for electrochemical sensing of $\mathrm{H}_{2} \mathrm{O}_{2}$. Microchim Acta 181:631-638. doi:10.1007/s00604-014-1164-2

20. Chen X, Wu G, Cai Z, Oyama M, Chen X (2013) Advances in enzyme-free electrochemical sensors for hydrogen peroxide, glucose, and uric acid. Microchim Acta 181:689-705. doi:10.1007/ s00604-013-1098-0

21. Yuping Lin XC (2015) Non-enzymatic sensing of hydrogen peroxide using a glassy carbon electrode modified with a nanocomposite made from carbon nanotubes and molybdenum disulfide. Microchim Acta. doi:10.1007/s00604-015-1517-5

22. Li D, Müller MB, Gilje S, Kaner RB, Wallace GG (2008) Processable aqueous dispersions of graphene nanosheets. Nat Nanotechnol 3:101-105. doi:10.1038/nnano.2007.451

23. Zöpfl A, Lemberger M-M, König M, Ruhl G, Matysik F-M, Hirsch $\mathrm{T}$ (2014) Reduced graphene oxide and graphene composite materials for improved gas sensing at low temperature. Faraday Discuss 173:403-414. doi:10.1039/c4fd00086b

24. Roddaro S, Pingue P, Piazza V, Pellegrini V, Beltram F (2007) The optical visibility of graphene: interference colors of ultrathin graphite on $\mathrm{SiO}_{2}$. Nano Lett 7:2707-2710. doi:10.1021/n10711581

25. Ferrari AC, Robertson J (2000) Interpretation of raman spectra of disordered and amorphous carbon. Phys Rev B 61:14095-14107. doi:10.1103/PhysRevB.61.14095

26. Ferrari AC, Meyer JC, Scardaci V, Casiraghi C, Lazzeri M, Mauri F, Piscanec S, Jiang D, Novoselov KS, Roth S, Geim AK (2006) 
Raman spectrum of graphene and graphene layers. Phys Rev Lett 97:187401. doi:10.1103/PhysRevLett.97.187401

27. Tuinstra F, Koenig JL (1970) Raman spectrum of graphite. J Chem Phys 53:1126-1130. doi:10.1063/1.1674108

28. Jin E, Lu X, Cui L, Chao D, Wang C (2010) Fabrication of graphene/prussian blue composite nanosheets and their electrocatalytic reduction of $\mathrm{H}_{2} \mathrm{O}_{2}$. Electrochim Acta 55:7230-7234. doi:10. 1016/j.electacta.2010.07.029

29. Li L, Du Z, Liu S, Hao Q, Wang Y, Li Q, Wang T (2010) A novel nonenzymatic hydrogen peroxide sensor based on $\mathrm{MnO}_{2}$ /graphene oxide nanocomposite. Talanta 82:1637-1641. doi:10.1016/j. talanta.2010.07.020

30. Liu S, Tian J, Wang L, Li H, Zhang Y, Sun X (2010) Stable aqueous dispersion of graphene nanosheets: noncovalent functionalization by a polymeric reducing agent and their subsequent decoration with Ag nanoparticles for enzymeless hydrogen peroxide detection. Macromolecules 43:10078-10083. doi:10.1021/ma102230m
31. Fu L, Lai G, Jia B, Yu A (2014) Preparation and electrocatalytic properties of polydopamine functionalized reduced graphene oxide-silver nanocomposites. Electrocatalysis 6:72-76. doi:10. 1007/s12678-014-0219-9

32. Maji SK, Sreejith S, Mandal AK, Ma X, Zhao Y (2014) Immobilizing gold nanoparticles in mesoporous silica covered reduced graphene oxide: a hybrid material for cancer cell detection through hydrogen peroxide sensing. ACS Appl Mater Interfaces 6: 13648-13656. doi:10.1021/am503110s

33. Tapas Kuila SB (2012) Chemical functionalization of graphene and its applications. Prog Mater Sci 57:1061-1105. doi:10.1016/j. pmatsci.2012.03.002

34. Heydrich S, Hirmer M, Preis C, Korn T, Eroms J, Weiss D, Schüller C (2010) Scanning raman spectroscopy of graphene antidot lattices: evidence for systematic p-type doping. Appl Phys Lett 97:043113. doi:10.1063/1.3474613 